\title{
Switchable Wide Tuning Range Bandstop Filters for Frequency-Agile Radios
}

\author{
Zhengzheng Wu, Yonghyun Shim, and Mina Rais-Zadeh \\ Department of Electrical Engineering \& Computer Science, University of Michigan, Ann Arbor, MI 48109, USA
}

\begin{abstract}
This paper reports on micromachined tunable bandstop filters that exhibit an octave frequency tuning range with high stopband rejection and low out-of-band loss. In addition to continuous frequency tuning, stopband switch on-off capability is realized by employing ohmic switches along with tunable capacitors. The fully re-configurable filters in this work are fabricated using a silicon-based integrated passive device technology and are the smallest-size bandstop filters reported in the low SHF range.
\end{abstract}

\section{Introduction}

Reconfigurable and cognitive radios require frequency-agile RF front-end modules to cover a wide range of wireless spectrum. Tunable filters are imperative components of cognitive radios and have been the focus of much research in the past [1], [2]. In the presence of strong out-of-band interferers, pre-select RF filters can protect the receiver from gain desensitization, and the receiver linearity requirement is significantly relaxed. Filters with high rejection provide $\mathrm{Tx} / \mathrm{Rx}$ isolation and are needed in frequency-division duplexing (FDD) transceivers and transmitter spurious emission control. Compared to bandpass filters, bandstop filters exhibit lower passband insertion loss, minimizing the degradation of receiver noise figure, while providing high rejection level for removing spurious signals. Therefore, tunable bandstop filters have the potential to replace more complicated and higher loss switched bandpass filter banks used in multi-band RF front-ends. In this paper, we report on a switchable wide tuning range bandstop filter. Filters are realized in a silicon-based integrated passive device (IPD) technology intended for multi-chip module integration.

\section{IPD Fabrication Process}

The silicon-based IPD technology developed in this work offers both high- $Q$ fixed RF passive components and tunable RF micro-electromechanical systems (MEMS) [3]. Fig. 1 shows the fabrication process steps. It offers three metal layers, one dielectric layer and a sacrificial layer. The process starts with passivating the substrate which is high resistivity $(>1 \mathrm{k} \cdot \mathrm{cm})$ silicon. The bottom metal is deposited and patterned. The sacrificial layer is then deposited and step etched to allow the formation of dual air-gaps (as shown in Fig. 1(d)) for realizing high analog tuning range ( $>5: 1)$ MEMS capacitors, and contact dimples used in MEMS ohmic switches. An electroplated gold layer is utilized as the structural layer for RF MEMS devices, and a thick electroplated copper layer is utilized for forming high- $Q$ inductive components and low-loss transmission lines. The sacrificial layer is removed to release the tunable capacitors and RF switches. Finally, silicon is selectively removed under inductive components to reduce the substrate loss.
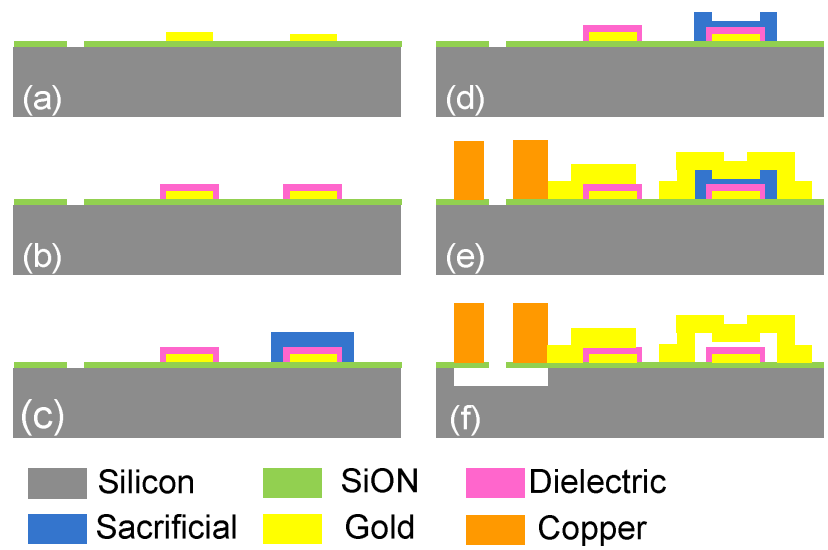

Fig. 1. Fabrication process flow of the IPD. (a): Deposit oxynitride dielectric as surface passivation layer and evaporate bottom metal layer; (b): Deposit and pattern dielectric layer for MIM capacitors; (c): Deposit a sacrificial layer; (d): Step etch the sacrificial layer; (e): Electroplate $2^{\text {nd }}$ metal layer for MIM capacitors and RF MEMS structural layer; (f): Electroplate thick metal for high- $Q$ inductors; (g): Sacrificial layer removal and substrate micromachining to create air-suspended inductors.

\section{Tunable Bandstop Filters}

The design of tunable bandstop filter is based on coupled transmission line configuration, as shown in Fig. 2. The transmission line is loaded with a capacitor for reduced electrical length. A pair of lumped coupled inductors (layout shown in Fig. 2) is used to imitate the coupled transmission lines in the band of interest. The advantage of using lumped components over transmission lines is that they occupy a smaller area. Using this filter design, narrow bandwidth bandstop filters with performances superior to conventional LC type filters can be implemented using low-value and high- $Q$ inductors. Wide tuning range MEMS capacitors are employed to tune the filter center frequency. As shown in Fig. 3, a fabricated MEMS capacitor can be tuned from 0.38 $\mathrm{pF}$ to $2.1 \mathrm{pF}$ with applying a voltage of up to $35 \mathrm{~V}$, demonstrating a tuning range of 5.5:1. The MEMS capacitor is modeled considering the resistive $\left(R_{\text {series }}\right)$ and inductive parasitic $\left(L_{\text {series }}\right)$, as shown in Fig. 4. Recalling from Fig. 2, the capacitor is connected in series with an inductor to form the resonator tank in the bandstop filter. The parasitic inductance of the capacitor $\left(L_{\text {series }}\right)$ is absorbed into the main inductor of the tank. Therefore, the overall $Q$ of the LC tank is improved and its usable frequency range is extended. 


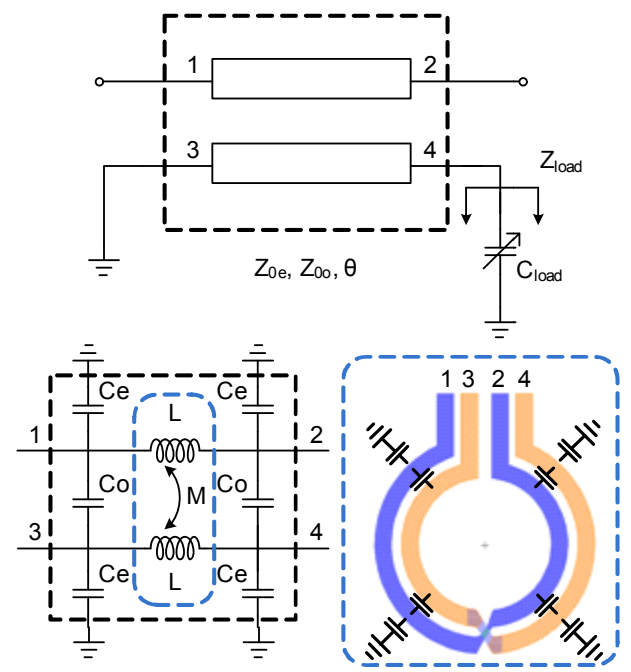

Fig. 2. Circuit implementation of the bandstop filter and layout of the lumped coupled inductor pair.

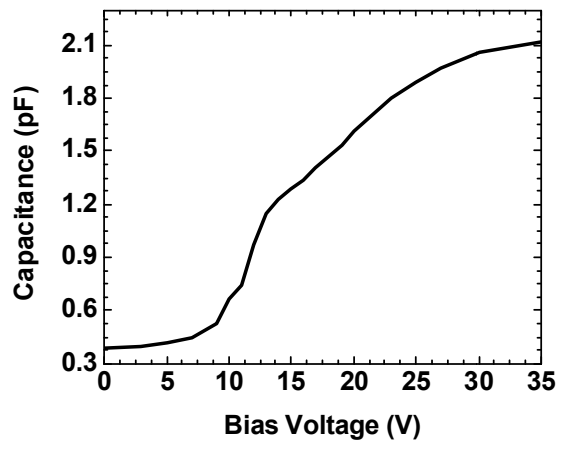

Fig. 3. Measured tuning range of a fabricated MEMS capacitor.

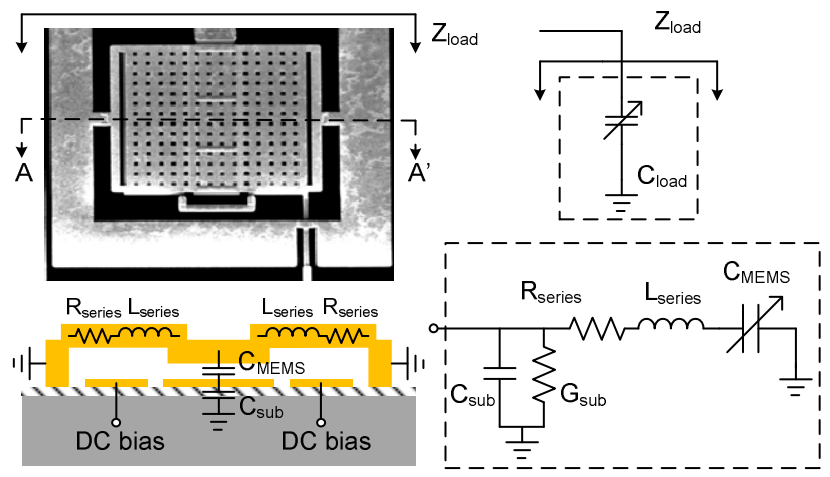

Fig. 4. Circuit model of the RF MEMS capacitor.

A two-pole tunable bandstop filter is realized by cascading two $1^{\text {st }}$-order bandstop filter cells. Fig. 5 shows a SEM image of a fabricated two-pole tunable bandstop filter. The size of the filter is $2.2 \mathrm{~mm} \times 2.6 \mathrm{~mm}$. The measured tuning results of the bandstop filter are plotted in Fig. 6. The center frequency is tuned from $6.5 \mathrm{GHz}$ to $3.1 \mathrm{GHz}$, achieving more than an octave frequency coverage. Across the entire tuning range, the insertion loss at both lower and higher passbands is measured to be less than $1 \mathrm{~dB}$. The filter shape shows significant improvement compared with other reported lumped LC filter implementations in this range [4][6]. The stopband rejection level is better than $20 \mathrm{~dB}$ in the frequency range of 5-6.5 GHz. At lower stopband center frequencies, the filter bandwidth is reduced as the capacitance of the tank is increased. Because of this bandwidth reduction and also the lower inductor $Q$ at low frequencies, the filter rejection level is degraded at frequencies below $5 \mathrm{GHz}$.

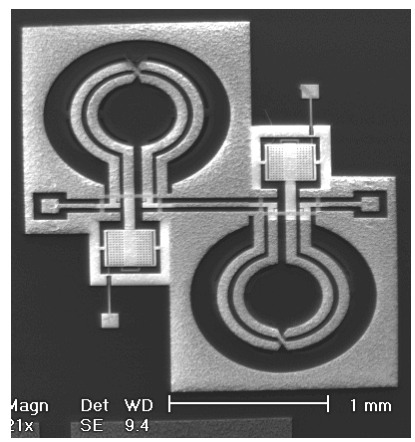

Fig. 5. A SEM image of a tunable bandstop filter.

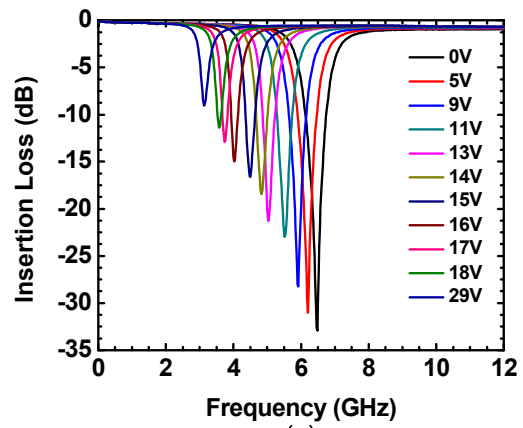

(a)

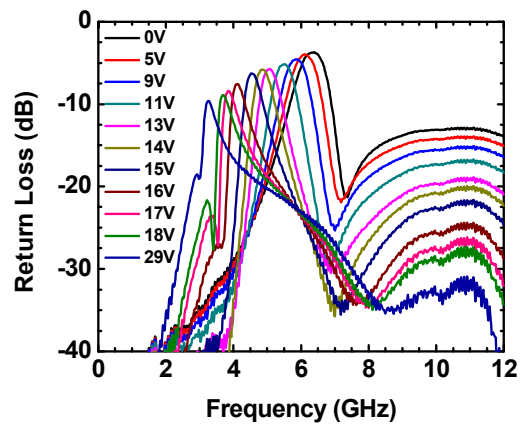

(b)

Fig. 6. Measured frequency response of the tunable bandstop filter (with bias voltage shown). (a) Insertion loss; (b) return loss.

The linearity of the tunable bandstop filter is characterized using a two-tone test. A two-tone input with center frequency of $5.25 \mathrm{GHz}$ and input power of $-10 \mathrm{dBm}$ is applied to the bandstop filter when the stopband is tuned to $5.25 \mathrm{GHz}$. Figs. 7(a) and (b) show the output spectrum with an input frequency offset of $1 \mathrm{kHz}$ and $1 \mathrm{MHz}$, respectively. 
The extracted $3^{\text {rd }}$-order input intercept point (IIP3) from the measurement is $3.6 \mathrm{dBm}$ at $1 \mathrm{kHz}$ frequency offset, as is shown in Fig. 8. At a higher two-tone frequency offset the $3^{\text {rd }}$-order inter-modulation output signals are below the noise floor, and the IIP3 cannot be extracted. This is because the MEMS capacitor has a lowpass response [1] that filters out the inter-modulation products with offset frequencies higher than the mechanical resonance frequency of the movable membrane. Therefore, inter-modulation products are highly attenuated if they fall in the lower and higher passbands of the bandstop filter, showing benefit of using MEMS devices as part of a filter.

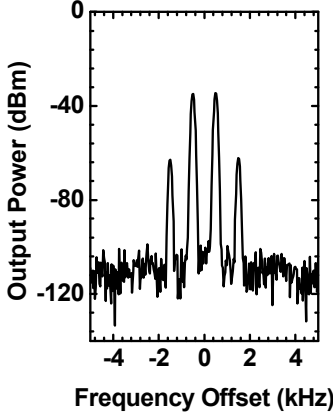

(a)

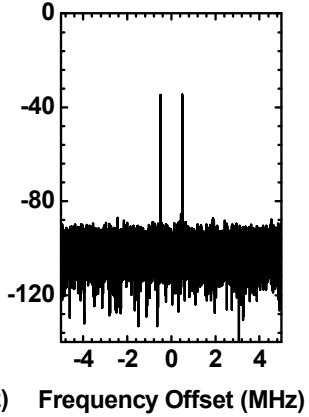

(b)
Fig. 7. Measured output spectrum with a two-tone test at $5.25 \mathrm{GHz}$ and -10 $\mathrm{dBm}$ input power; (a) $1 \mathrm{kHz}$ offset, and (b) $1 \mathrm{MHz}$ offset.

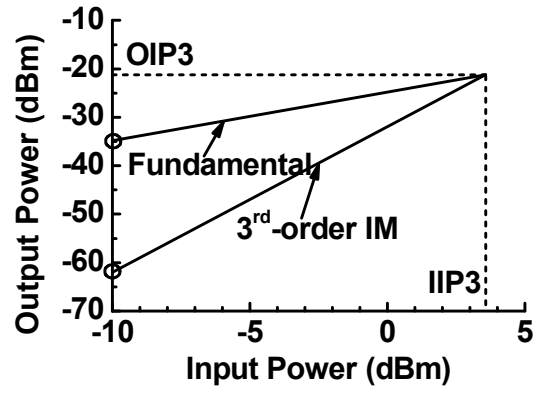

Fig. 8. Extraction of IIP3 with a two-tone input frequency offset of $1 \mathrm{kHz}$.

The tuning speed of the bandstop filter is measured by applying a step-function actuation bias to the MEMS capacitors. The measured tuning speed of the tunable bandstop filter is better than $80 \mu$ s as shown in Fig. 9.

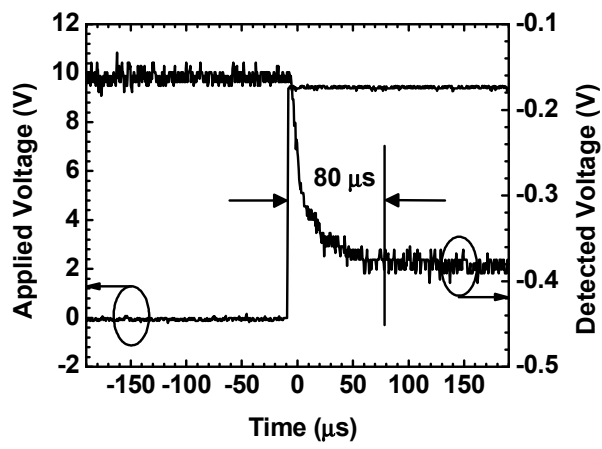

Fig. 9. Measured tuning speed of the tunable bandstop filter. The tuning speed is better than $80 \mu \mathrm{s}$.

\section{Switchable Bandstop Filters}

In a cognitive spectrum utilization scheme, it is useful to switch off the bandstop filter in case no interference is present. When the filter is switched off, additional channel loss introduced by the filter will be minimized and the available wireless spectrum will be fully utilized. In this work, a MEMS ohmic switch is exploited to add switch on/off capability to the wide tuning range bandstop filters. As shown in the circuit schematic of Fig. 10, a MEMS ohmic switch is connected in parallel with the tunable capacitor. When the RF MEMS ohmic switch is in contact, it shorts the capacitive load port to the ground. In this switched-off state, the filter becomes an all-pass network.

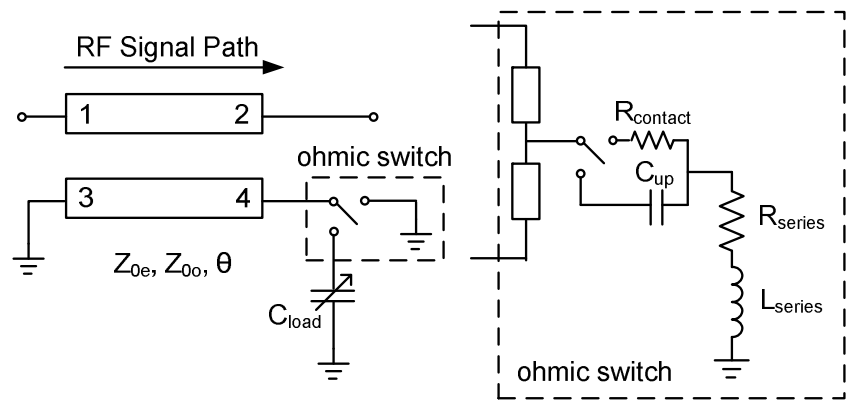

Fig. 10. Circuit model of the tunable bandstop filter with switch on/off capability and the electrical model of the MEMS ohmic switch.

In the proposed switchable bandstop filter design, the MEMS switch is placed in the coupled line section instead of in the main RF signal path (Fig. 10). Therefore, the filter loss is less sensitive to the contact resistance of the ohmic switch. The pass-band insertion loss of the filter having different contact resistance $\left(R_{\text {contact }}\right)$ for the switch is simulated and shown in Fig. 11. Simulation results indicate that even with a contact resistance of $10 \Omega$, the insertion loss is less than $0.83 \mathrm{~dB}$ up to $10 \mathrm{GHz}$. Because of the low sensitivity to contact resistance, the switchable filter can also employ RF MEMS switches that use hard metals (e.g. Ru) as the contact material. Such switches offer good reliability [7].

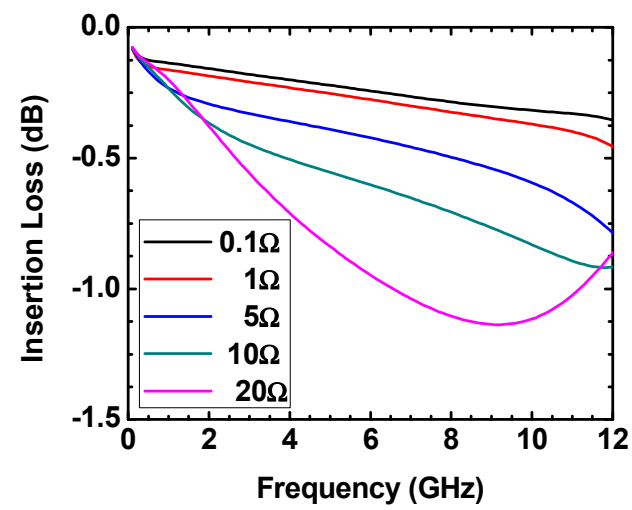

Fig. 11. Insertion loss of the filter path when the bandstop filter is switched off using a MEMS ohmic switch. 
A SEM image of a fabricated switchable filter is shown in Fig. 12. The RF MEMS ohmic switch is embedded in the capacitive load port, as can be seen in the close-up view show in Fig. 12. The size of the switchable filter is $2.2 \mathrm{~mm} \times$ $2.6 \mathrm{~mm}$, similar to the size of the tunable bandstop filter. The measured response of the filtering path when the bandstop filter is switched off is shown in Fig. 13. With increasing actuation voltage on the switch, the contact resistance of the RF MEMS switch is reduced and the insertion loss of the switched-off bandstop filter is improved. The measured insertion loss is less than $0.84 \mathrm{~dB}$ up to 10 $\mathrm{GHz}$. The higher insertion loss in measured results is due to the higher resistance of the electroplated metal and fabrication imperfections that cause non-ideal electrical performance for the lumped coupled inductors. The bandstop filter responses at switch on/off states are compared in Fig. 14.

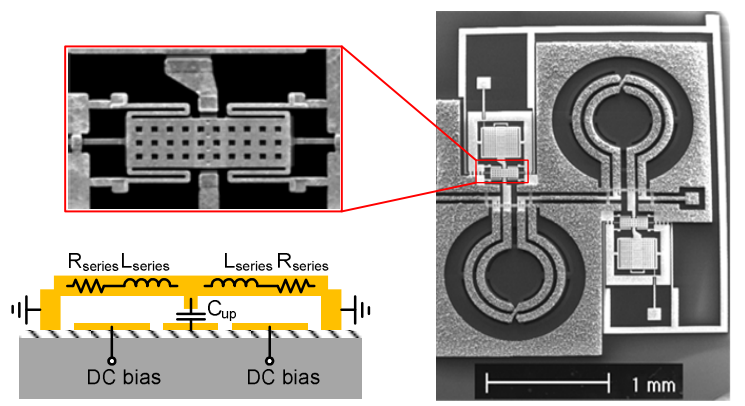

Fig. 12. A SEM image of the fabricated tunable bandstop filter with closedup view and circuit model of the RF MEMS ohmic switch (up-state).

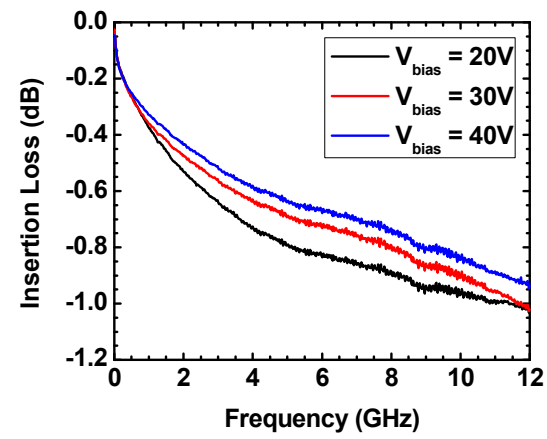

(a)

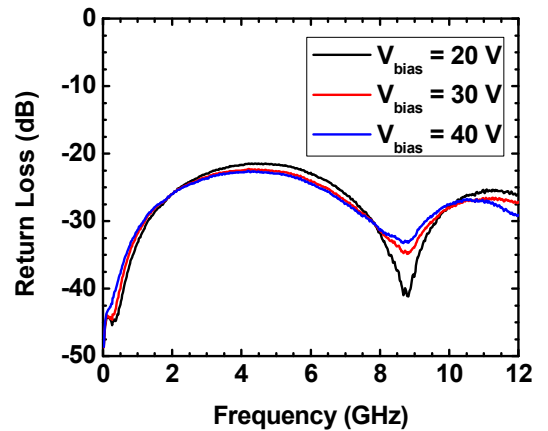

(b)

Fig. 13. Measured responses of the switched-off bandstop filter with varied DC bias on MEMS ohmic switch; (a) Insertion loss, and (b) return loss.

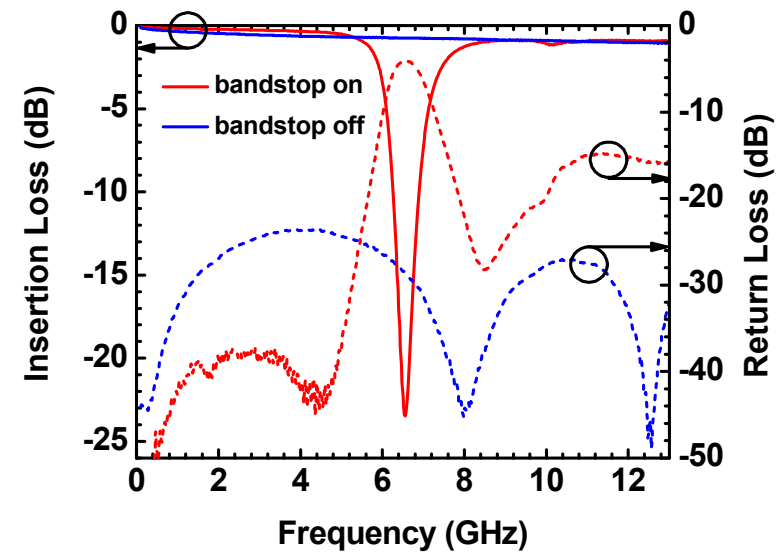

Fig. 14. Insertion loss and return loss when the bandstop filter is switchedon and switched-off (a $30 \mathrm{~V}$ bias on ohmic switch).

\section{Conclusion}

In this paper, wide tuning range miniaturized bandstop filters are implemented. These tunable filters provide high interference rejection capability and low passband insertion loss. A stopband switch on-off technique is proposed which is tolerant to contact resistance of ohmic switches. Filters are fabricated using a silicon-based IPD technology which can be used to realize other miniaturized frequency-agile RF front-ends.

\section{Acknowledgement}

The authors would like to acknowledge the staff of the Lurie Nanofabrication Facility at the University of Michigan for their assistance with fabrication. This work is supported by National Science Foundation under award number1055308.

\section{References}

[1] G.M. Rebeiz, et al., "Tuning in to RF MEMS," IEEE Microw. Mag., vol. 10 , no. 6 , pp. $55-72$, Oct. 2009.

[2] H.A.C. Tilmans, W. De Raedt, and E. Beyne, "MEMS for wireless communications: 'from RF-MEMS components to RF-MEMS-SiP'," Journal of Micromechanics and Microengineering, vol. 13, pp. S139163, July 2003.

[3] Z. Wu, Y. Shim, and M. Rais-Zadeh, "Miniaturized UWB bandpass filters integrated with notch filters using a silicon-based integrated passive device technology," 2011 IEEE MTT-S International Microwave Symposium, June 2011.

[4] M. Fernandez-Bolaos, T. Lisec, C. Dehollain, D. Tsamados, P. Nicole, and A.M. Ionescu, "Highly tunable band-stop filters based on AIN RF MEM capacitive switches with inductive arms and zipping capacitive coupling," 2009 IEEE International Electron Devices Meeting, Dec. 2009.

[5] P. Ekkels, et al., "Air gap-based MEMS switch technology using nickel surface micromachining," Sensors and Actuators A: Physical, vol. 166, no. 2, pp. 256-263, Apr. 2011.

[6] H.S. Lee, D.H. Choi, and J.B. Yoon, "MEMS-based tunable LC bandstop filter with an ultra-wide continuous tuning range," IEEE Microw. Wireless Compon. Lett., vol. 19, no. 11, pp. 710-712, Nov. 2009.

[7] C.D. Patel and G.M. Rebeiz, "An RF-MEMS switch with $\mathrm{mN}$ contact forces," 2010 IEEE MTT-S International Microwave Symposium, May 2010. 